\title{
EchoGéo
}

19 | 2012

Du littoral à la haute mer : quelles recherches récentes en géographie?

\section{Entretien avec Fabien Hennion}

Maria Gravari-Barbas et Sébastien Jacquot

\section{(2) OpenEdition \\ Journals}

Édition électronique

URL : https://journals.openedition.org/echogeo/13005

DOI : 10.4000/echogeo.13005

ISSN : 1963-1197

Éditeur

Pôle de recherche pour l'organisation et la diffusion de l'information géographique (CNRS UMR 8586)

Référence électronique

Maria Gravari-Barbas et Sébastien Jacquot, « Entretien avec Fabien Hennion », EchoGéo [En ligne], 19 | 2012, mis en ligne le 10 février 2012, consulté le 11 août 2021. URL : http://journals.openedition.org/ echogeo/13005; DOI : https://doi.org/10.4000/echogeo.13005

Ce document a été généré automatiquement le 11 août 2021.

EchoGéo est mis à disposition selon les termes de la licence Creative Commons Attribution - Pas d'Utilisation Commerciale - Pas de Modification 4.0 International (CC BY-NC-ND) 


\title{
Entretien avec Fabien Hennion
}

\author{
Maria Gravari-Barbas et Sébastien Jacquot
}

1 Après un premier poste dans Cabinet d'études (Détente Consultants), Fabien Hennion est, depuis 2010, chargé de mission à l'Union Départementale des Offices de Tourisme et Syndicats d'Initiative du Nord (UDOTSI).

2 - Maris Gravari-Barbas (MGB), Sébastien Jacquot (SJ). Quel cheminement vous a-t-il permis de passer des études de géographie au tourisme?

3 - Fabien Hennion (FH). Après l'obtention de mon baccalauréat scientifique je me suis tourné vers la géographie, le développement et l'aménagement des territoires. J'ai d'abord suivi un cursus de 3 ans au sein de l'Institut d'Aménagement et d'Urbanisme de Lille (IAUL), rattaché à l'Université Lille 1. J'ai obtenu une licence en Géographie Développement et Aménagement des Territoires. Après ces années lilloises, j'ai choisi de me spécialiser dans l'aménagement et le développement touristique des territoires. J'ai donc poursuivi ma formation en faisant le Master «Développement et Aménagement Touristique des Territoires" à l'Institut de Recherche et d'Etudes Supérieures sur le Tourisme (IREST) rattaché à l'Université Paris 1 Panthéon Sorbonne. J'y ai obtenu un Master professionnel dans ma spécialité, en 2009.

4 Ma dernière année de formation a été l'occasion d'un stage de 6 mois dans un bureau d'études parisien spécialisé en ingénierie touristique. Suite à ce stage il m'a été proposé d'intégrer l'équipe de cette société en tant que chargé d'études, j'y ai donc signé un CDI en septembre 2009. Après 1 an en poste j'ai eu l'opportunité d'évoluer dans mon métier en rejoignant l'Union Départementale des Offices de Tourisme et Syndicats d'Initiative $\mathrm{du}$ Nord (UDOTSI) qui recrutait alors un chargé de mission autour de la mise en place et de l'animation du «Plan d'actions 2007-2013 du réseau des OT-SI du Nord». Aujourd'hui je travaille notamment autour de la mise en place de Schémas Locaux de Développement Touristique, de l'enjeu de la durabilité via l'animation d'un Agenda 21, de la professionnalisation du réseau départemental des offices de Tourisme, ...

5 - MGB, SJ. En quoi votre formation de géographe vous a-t-elle permis de faire votre métier actuel ? Que vous a-t-elle apporté en termes de compétences dans le tourisme?

6 - FH. Pour moi, la géographie a toujours été synonyme de polyvalence. Tout au long de ma formation, c'est cet aspect qui m'a particulièrement motivé. Etudier l'espace, le 
territoire, conduit nécessairement à s'intéresser à une multitude de disciplines, toutes liées à ce champ d'étude. Des sciences dures aux sciences humaines, des risques " naturels » à la géopolitique, tout semble trouver un sens et une corrélation quand il s'agit d'appréhender les territoires. Cet aspect très transversal a toujours orienté mes choix de formation. Ma spécialisation vers le champ d'étude du tourisme est liée à cela, cette transversalité me paraissait encore présente dans ce secteur. On entend souvent dire qu'il s'agit justement de formations "généralistes ", " peu spécialisées ", pour moi ces critiques ont au contraire toujours été un plus... Et même un objectif !

7 A l'entrée sur le marché du travail j'ai pu constater que cette polyvalence était plutôt recherchée. Ma formation me permet ainsi d'avoir une culture générale sur le champ touristique, les questions liées aux territoires et à l'espace mais également la capacité de mobiliser certains outils et méthodologies propres à la pensée géographique.

8 - MGB, SJ. Pensez-vous que la pensée géographique structure votre approche professionnelle?

9 - FH. Dans mon travail, dans les tâches quotidiennes comme dans les projets plus longs, j'ai tendance à relier l'ensemble des questions que je me pose à des notions de territoires. Bien sûr, le lien apparaît plus ou moins directement, mais d'une manière générale l'approche des questions par des notions spatiales me semble apporter un plus. Au niveau des Offices de Tourisme, quand je m'intéresse au fonctionnement de telle ou telle structure, les considérer comme faisant partie d'un système d'acteurs, implantés dans un contexte territorial spécifique permet souvent d'expliquer certaines choses. Quand cette manière de penser est possible il me semble que ça apporte un réel éclairage à certaine situation. Ce n'est pas une démarche formalisée, elle apparaît plutôt en fil rouge.

Quand on parle de développement touristique, on est parfois confronté à des oppositions entre la notion de "destination touristique ", un territoire cohérent et homogène pour le touriste, et le «territoire institutionnel ", où une compétence donnée s'exerce. On est bien là au cœur de problématiques spatiales qui influent sur des systèmes complexes. Dans ces cas-là la pensée géographique me semble une approche particulièrement pertinente pour concilier au mieux l'ensemble.

11 - MGB, SJ. Votre formation de géographe vous a-t-elle donc permis de vous approprier mieux, ou différemment les différents dossiers sur lesquels vous travaillez actuellement?

12 - FH. On en revient aux notions de polyvalence que j'abordais précédemment. Ce besoin de toucher à de nombreuses disciplines et de comprendre des sujets très divers entraîne nécessairement le besoin de développer une certaine "curiosité ». A mon sens, la formation de géographe permet d'aiguiser cette curiosité et de donner des clés méthodologiques, des outils, pour résoudre d'éventuels problèmes d'incompréhension, des blocages face à l'inconnu.

13 Peut-être que j'interprète mal les choses, et qu'il faut plutôt les prendre à l'envers, à savoir que ce sont des profils naturellement « curieux » qui se dirigent vers le champ de la géographie et alimentent ces formations... En tout cas aujourd'hui, dans les tâches qui m'incombent, j'essaie de garder toujours cette envie de découvrir la nouveauté, sans me cantonner à ce que je connais déjà. Ma formation est en partie responsable de cela. 
14 Par exemple, la question du numérique et de l'e-tourisme est au cœur de nombreuses problématiques depuis mon entrée dans le monde professionnel. Que ce soit en bureau d'études ou maintenant à l'UDOTSI, c'est un sujet qui fait partie de mes préoccupations parce qu'il intéresse les structures du tourisme et les territoires. C'est à la fois un sujet qui ne cesse d'évoluer, c'est d'ailleurs là tout l'enjeu, et un sujet qui ne m'était pas familier. La curiosité déjà évoquée m'a permis de m'y intéresser et de mieux en cerner les tenants et les aboutissants. On est peut-être là assez éloigné de la géographie, encore que..., mais de toute évidence on retrouve des questions d'organisation, de système d'acteurs, d'échanges, reliées à la discipline.

15 Je pourrais également appliquer ce raisonnement à la thématique du développement durable. L'UDOTSI du Nord ayant élaboré et animant un Agenda 21, c'est un sujet central dans mon travail. Sans revenir en détail sur cette problématique particulièrement porteuse d'enjeux aujourd'hui dans le tourisme, là encore la géographie permet d'aborder ces questions de manière pertinente. En effet, que ce soit pour un office de Tourisme, une collectivité, une structure touristique, son ancrage spatial est une clé d'entrée fondamentale pour un développement plus réfléchi.

16 - MGB, SJ. Votre formation de géographe est-elle reconnue et appréciée par votre employeur?

17 - FH. Même si ma formation de géographe n'a peut-être pas été le déclencheur de mes recrutements, je pense que c'est certainement un plus aujourd'hui, notamment parce que le monde du tourisme est confronté à des réformes et des changements que l'œil du géographe peut appréhender différemment.

18 Les réformes touchent les territoires, ils évoluent, vont encore évoluer, les structures touristiques sont impactées. On en revient aux notions développées précédemment, de prise en compte du contexte spatial dans l'organisation des systèmes d'acteurs. Pour moi on est bien au cœur de la géographie.

19 - MGB, SJ. Dans l'exercice de votre métier, établissez-vous des passerelles entre votre emploi et la recherche appliquée?

20 - FH. Je me souviens m'être posé cette question lors de la rédaction de mon rapport de stage, suite à ma première immersion de 6 mois en bureau d'études. Et cet exercice m'avait alors permis de dépasser certains de mes a priori qui me faisaient difficilement relier ces deux mondes. A l'époque j'en avais conclu que ces deux univers, bien éloignés en apparence, se nourrissaient finalement mutuellement l'un de l'autre. Les acteurs du monde professionnel se nourrissent du monde de la recherche, cela permet alors à ce dernier d'observer des utilisations et applications concrètes de la connaissance produite. Dans mon travail, côté professionnel donc, cela se traduit par exemple par une veille autour de certaines sources et de certains auteurs aperçus pendant ma formation universitaire, qui continuent d'alimenter parfois mon travail.

21 Par ailleurs, il me semble qu'un parallèle peut également être fait en termes de méthode. D'une manière générale, si l'on résume la recherche, de manière très basique, à la chaîne suivante : Problématiser une question / Emettre des hypothèses / Répondre à ces hypothèses par une méthodologie adéquate / Amener d'autres questionnements, on se rend compte que le même schéma peut s'appliquer à l'ensemble des tâches qui animent les missions d'un professionnel. Au quotidien on est parfois loin de la rigueur scientifique mais la structuration de la pensée suit le même enchaînement. En prenant 
un peu de recul et en analysant l'ensemble des tâches qui font mon métier, je constate que cette comparaison peut souvent se faire. fréquemment. Les outils issus de ma formation sont à l'image de mes études, assez diversifiés ; pas uniquement liés à la géographie au sens strict, mais plus à sa dimension de science humaine. Il est souvent question d'analyse qualitative, voire quantitative, dans certaines de mes tâches. Les outils de traitement statistique peuvent alors être utilisés. De même, pour avancer dans n'importe quel projet, il est nécessaire de recourir à l'écoute d'autres acteurs liés au sujet. Les techniques d'entretiens, d'interviews, d'enquêtes, sont alors sollicitées. La lecture critique, la synthèse, la communication orale sont également ancrées dans mon travail. Là encore il s'agit d'outils et de méthodes développés tout au long de ma formation. Quand on aborde la géographie, on pense souvent aux outils de cartographie. Aujourd'hui ce sont peut-être les techniques que j'utilise le moins au quotidien. Pas par manque d'intérêt, au contraire, mais plutôt parce qu'il s'agit de techniques particulièrement chronophages qui font l'objet de métiers à part entière et auxquels on peut avoir recours. Les SIG m'ont par exemple beaucoup intéressé durant mes études, mais se retrouvent beaucoup moins dans mes tâches actuelles. Dans tous les cas, l'apprentissage et l'utilisation de ces outils favorisent d'une certaine manière ma compréhension des représentations spatiales, qui apportent un réel plus dans toute analyse, comme je l'évoquais précédemment.

EchoGéo, 19 | 2012 